Membranen, die ebenfalls Di-Bazillen ergeben. Am 17. Oktober Injektion von 1000 I.-E. Heilserum. Das Kind ist jetzt sehr elend, der Puls außerordentlich schlecht, und man ist genötigt, Kampfer und Koffein zu geben. Das Erbrechen, das in den letzten Tagen zwar sehr häufig, aber viel weniger heftig war, hört nun plötzlich ganz auf; trotzdem sinkt das Gewicht bis zum 21. Oktober um $400 \mathrm{~g}$, da die RingerInfusionen eingestellt werden müssen, weil sie sehr schlecht resorbiert werden und außerdem Oedeme an Beinen und Füßen sich zeigen. Nun das Erbrechen aufgehört hat, wird versucht, dem Kinde stündlich $50 \mathrm{~g}$ Abgedrückte zu geben. Die Milch- sowie die Breimenge werden nach und nach gesteigert. Dies gelingt sehr gut, es erfolgt kein Erbrechen; das Gewicht steigt trotz des schlechten Allgemeinbefindens. Die Temperatur, die sich seit dem 14 . Oktober auf $37,5^{\circ}$ gehalten hat, steigt am 25. Oktober auf $38,6^{\circ}$. Am 26. Oktober sind die Oedeme ganz zuruckgegangen, das Gewicht ist trotzdem wieder $420 \mathrm{~g}$ hoher als am 21. Oktober. Die Temperatur ist an diesem Tage $\mathbf{3 6 , 4 ^ { 0 }}$.

Vom 27. Oktober ab, wo das Kind vollständig entfiebert, der Puls wieder gut, das Gewicht auf $3820 \mathrm{~g}$ gestiegen ist, beginnt das Erbrechen von neuem, anfangs selten und wenig, dann wieder öfter und heftiger, schließlich fast nach jeder Flasche.

Am 6. November ist die Temperatur 37,5 ${ }^{\circ}$. Die pflegende Schwester hat den Eindruck, als ob das Kind heute öfter erbricht als sonst, weil es beim Trinken husten muß und sich deswegen verschluckt, doch ist dariber nichts Bestimmtes zu sagen. Die sofortige Untersuchung ergibt Bronchopneumonie beider Lungen. Am nächsten Tage immer wieder geringes Erbrechen durch Verschlucken. Dann aber, mit der steigenden Versehlechterung des Befindens, sistiert das Lrbrechen, obschon das Kind bis fast zum Ende gut trinkt, vollig. Exitus am 17. November 1913.

Die Obduktion ergab neben dem spezifischen Befunde an der (Direktor: Dr. J. Ritter.)

\title{
Ein Beitrag zu den nervösen Aequivalenten im Säuglingsalter.
}

\section{Von Margarete Wilhelm, Medizinalpraktikantin.}

Vor einigen Wochen starb hier im Säuglingskrankenhaus an einer auf eine echte Nasendiphtherie folgenden Pneumonie ein achtmonatiger Säugling, dessen eigentümliches Verhalten während der zwei kurz hintereinander folgenden Krankheitsepochen uns sehr auffiel.

Das Kind, J. E. J., geboren am 14. März 1913, wurde uns am 26. Oktober 1913 aus einem Hein in Pankow zugeschickt, weil es trotz Brusternährung seit einiger Zeit nicht vorwärts kommen wollte und täglich mehrere Male erbrach. Es stammte von einer sehr nervösen Mutter. Ueber den Vater wissen wir nichts Näheres; ebensowenig waren nähere anamnestische Angaben über das Kind zu erhalten, das nach Aussage der Mutter bei der Geburt voll und rund war, jetzt aber blaß und elend aussah.

Status bei Einlieferung des Kindes ins Säuglingskrankenhaus am 26. Oktober 1913: Kind in schlechtem Ernährungszustande; Wangen eingefallen. Gesicht lang, Kinn spitz, Augen lebhaft. Fettpolster und Muskulatur kaum vorhanden. Turgor der Haut sehr reduziert. Starker Intertrigo am Gesäß. Schädelumfang $38,5 \mathrm{~cm}$; Lànge $61 \mathrm{~cm}$. Halsvenen treten beim Schreien stark hervor. Fontanelle offen. Nirgends Drüsen. Puls von guter Füllung und Spannung. Cor innerhalb normaler Grenzen. Tóne rein. Pulmones: überall voller Lungenschall, reines pueriles Atmen. Abdomen eingesunken; Bauchdecke schlaff. Diastase der Mm. recti. Leib weich, keine Organvergrößerungen. Reflexe: Pupillen reagieren. Patellarreflexe normal. Aufnahmegewicht: $3730 \mathrm{~g}$. Aufnahmetemperatur: 36,50 .

Vom Tage der Aufnahme an erhält das Kind, das ungefähr die Hälfte des Sollgewichtes hat, abgedrückte Milch in kleinen Mengen. Es speit und erbricht nach jeder Mahlzeit und kommt immer mehr herunter. Magenspülung und längere Zeit hintereinander gegebene Dosen von Brom haben keine Wirkung, ebensowenig Novokain oder Erepton. Am 15. September, drei Wochen nach der Einlieferung, ist das Gewicht des Kindes auf $3570 \mathrm{~g}$ gesunken. Da es sehr elend ist und, ordentlich austrocknet", ist man genötigt, Ringer-Infusionen und Nährklistiere zu verabreichen. Nun wird neben der Flasche Brei gegeben, den das Kind behält, während es nach der Flasche immer noch stark schüttert. Dabei steigt das Gewicht innerhalb acht Tagen um $580 \mathrm{~g}$. Eine am 23. Oktober eintretende Verdauungsstörung und erneutes, noch heftigeres Erbrechen als fruher bedingen wieder ein starkes Sinken des Gewichtes, das am 10. Oktober nur noch $3410 \mathrm{~g}$ beträgt. Die Temperatur ist an diesem Tage $35,6^{\circ}$. Nun ist man wieder auf ganz kleine Mengen abgedrückter Milch angewiesen; außerdem werden Teeinläufe und RingerInfusionen gemacht. Am 11. Oktober hat das Kind etwas Schnupfen; Temperatur $36,4^{0}$. Am 13. Oktober ist der Schnupfen stärker geworden; die Nase sondert fleischwasserähnliches Sekret ab. Nasenabstrich, Kulturund Tierversuch bestätigen die Diagnose: Nasendiphtherie. Inzwischen ist auch das linke Auge stark entzündet und geschwollen. Es finden sich Lunge einen antrumkontrahierten Magen. Die Pylorusmuskulatur war nur wenig verdickt. Eine Sonde von etwa $1 \frac{11}{2} \mathrm{~mm}$ Durchmesser geht glatt durch den Pylorus hindurch.

Während es zu Lebzeiten oft nicht möglich ist, palpatorisch eine echte Pylorusstenose vom Pylorospasmus auf nervöser Grundlage zu unterscheiden, ist hier durch die Obduktion der Beweis geliefert, daß keine anatomische Ursache für das Verhalten des Kindes Monate hindurch besteht. Eine Stenose des Pylorus ist nicht vorhanden, ebensowenig eine stärkere Hypertrophie der Pylorusmuskulatur, noch eine Veränderung der Magenschleimhaut. Die geringe vorhandene Hypertrophie der Pylorusmuskulatur kann man leicht dadurch erkJären, daß oft in Tätigkeit versetzte Muskeln meist hypertrophieren.

Wie aber hat sich das ganz gewohnheitsmäßige Erbrechen herausgebildet, wenn keine anatomische Anomalie des Magens besteht? Lust nimmt eine Motilitätsneurose an und bezieht sich dabei auf Pawlows und Krasnogorskis Experimente, besonders aber auf Ibrahims Arbeit: Ueber pathologische Bedingungsreflexe. - Daß für das in den ersten Lebensmonaten eingetretene Erbrechen zuerst eine Ernährungsstörung die Ursache war, ist nicht nur anzunehmen, sondern als erwiesen zu betrachten. Aus der sehr mangelhaften Anamnese können wir ersehen, daß das Kind, das nach Aussage der Mutter bei seiner Geburt ,,voll und rund" war, von einer späteren, nicht genau bekannten Zeit ab erbrach und nicht vorwärts kommen wollte, im Gegenteil abnahm und bei der Einlieferung ins Krankenhaus weniger als sein Geburtsgewicht aufwies. Außerdem ist das Erbrechen an und für sich - da keine nachweisbare Pylorusstenose vorhanden war - als ein Beweis für eine einmal vorhandene Ernährungsstörung anzusehen. Die Berührung der Speisen mit der gesunden Magenschleimhaut löst sekretorische und gewisse motorische Tätigkeiten des Magens aus, die darauf gerichtet sind, zuerst Magensaft zu sezernieren und dann den produzierten Magensaft mit den eingeführten Speisen zu vermischen; es werden unbeding te sekretorische und motorische Reflexe ausgelöst, ,, unvermeidliche Folgen gewisser Reize unter beliebigen Bedingungen". (Pawlow, Krasnogorski.) Ebenso als unbedingter Reiz wirkt auf die erkrankte Magenschleimhaut die Berührung mit zugeführten Speisen; es werden motorische Reflexe ausgelöst, die antiperistaltische Magenbewegungen verursachen und die zugeführten Speisen auf umgekehrtem Wege möglichst rasch wieder aus dem Magen hinausbefördern und die überempfindliche Magenschleimhaut von der ihr unangenehmen Berührung befreien.

Nach Pawlows Versuchen an Hunden und Krasnogorskis Experimenten mit Kindern kann aber jeder unbedingte Reflex in einen bedingten verwandelt werden, 
,,indem irgendein indifferenter Reiz zeitlich einige Male mit einem unbedingten Reiz und der dadurch hervorgerufenen reflektorischen Tätigkeit zusammentrifft". Für die Pawlowschen Hunde z. B. wurde das Geräusch eines Metronoms dadurch zum bedingten Reiz, die Sekretion des Magensaftes zum bedingten Reflex, daß das Metronomgeräusch zeitlich einige Male mit dem Füttern zusammenfiel. Bei Kras nogorskis Versuchen wurde die Mundbewegung des Versuchskindes zum bedingten Reflex, das Metronomgeräusch zum bedingten Reiz dadurch, daß eine Schokoladegabe eine Zeitlang immer mit dem Metronomgeräusch verabreicht wurde. Die Tätigkeit der Speicheldrüsen bei den Hunden und das Mundöffnen der Kinder wurden mit dem indifferenten Reize ,Metronomgeräusch" in zeitlichen Zusammenhang gebracht.

Ebenso ruft schließlich schon allein der Anblick einer anwidernden Substanz die ein- oder einige Male durch direkte Reizung der Mundschleimhaut mittels dieser Substanz hervorgebrachte Speichelsekretion hervor.

Uebertragen wir diese Erfahrungen auf unseren Fall, so kann ganz wohl bis żu dem Moment, da die Magenschleimhaut wieder gesundet und ihre Ueberempfindlichkeit, die das erste und die folgenden Male Erbrechen auslöste, geschwunden ist, der unbeding te Reflex zu einem bedingten geworden sein, indem das Anfüllen des Magens, das Saugen oder gar der bloße Anblick der Flasche, irgendein indifferenter Reiz zu einem bedingten, antiperistaltische Bewegungen auslösenden geworden ist, denn: "Dấ erste charakteristische Merkmal der bedingten Reflexe bei Kindern ist die außerordentliche Schnelligkeit der Bildung"; doch ist die Bildung der bedingten Reflexe ebenso wie das Erlöschen der einmal gebildeten bedingten Reflexe ,,bei verschiedenen Kindern verschieden". Krasnogorski fand, daß sie bei normalen Kindern ebenso leicht erlöschen, wie sie entstehen; daß sie bei manchen neuropathischen schwer entstehen und dabei äußerst leicht erlöschen, bei anderen wieder sehr leicht entstehen und schwer erlöschen; bei normalen Kindern braucht also der indifferente Reiz nur kurze Zeit mit dem unbedingten zusammenzufallen, um ein bedingter zu werden und einen bedingten Reflex auszulösen. Der bedingte Reflex aber wird auch sehr bald erlöschen, wenn der indifferente Reiz nur wenige Male ohne den unbedingten gewirkt hat.

Das Erlöschen ist in diesem Falle von der längeren oder kürzeren Abwesenheit des direkten Reizes abhängig. Es kann aber auch dadurch bedingt werden, daß zu dem indifferenten Reiz, z. B. Metronomgeräusch bei den Pawlowschen Hunden, der seither einen sekretorischen Bedingungsreflex ausgelöst hat, plötzlich ein dritter, z. B. Licht, gefügt wird. Das Licht wird, wenn es einige Male mit dem Metronomgeräusch zusammengewirkt hat und dabei keine Nahrungsgabe erfolgt ist, schließlich als Hemmnis wirken, es wird keine Speichelabsonderung mehr erfolgen.

Dürfen wir diese Gesetze auf unseren Fall anwenden, so wird das unstillbare, monatelang fortdauernde und plötzlich mit dem Eintreten einer schweren Erkrankung aufhörende Erbrechen bei einem von einer sehr nervösen Mutter stammenden Kinde ganz wohl dadurch erklärt, daß man annimmt, daß bei einem neuropathischen Kinde, bei dem früher einmal eine von Erbrechen begleitete Ernährungsstörung bestanden hat, sich ein bedingter Reflex herausgebildet hatte, der monatelang anhielt und erst dann ganz plötzlich erlosch, als eine schwere Erkrankung, die sozusagen als Hemmnis wirkte, eintrat. Mit dem Verschwinden der Krankheit stellte sich der Bedingungsreflex wieder-ein, um bei erneutem Hinzutreten siner gleichartigen Hemmung wieder zu erlöschen. 